\title{
Desarrollo, análisis y evaluación de la producción oral en clase de lengua extranjera con las nuevas tecnologías
}

\author{
Mario TOMÉ DíEz \\ Universidad de León \\ Departamento de Filología Moderna \\ mtflenet@yahoo.es
}

Recibido: $16 / 03 / 2015$

Aceptado: 15/09/2015

\begin{abstract}
Resumen
En este estudio experimental se presentan herramientas y métodos que permiten desarrollar la competencia de producción oral entre alumnos universitarios que cursan una asignatura de francés lengua extranjera. Se realiza una medición de la producción oral de los alumnos basada en el análisis de la duración del tiempo real de grabaciones en podcasts, así como en la estimación de los alumnos a partir de un autoinforme sobre su producción oral. Se proponen varios criterios para evaluar las producciones orales en relación con la corrección de la pronunciación (repetición, autorreflexión, método, colaboración) quedando todos ellos recogidos en las grabaciones audio de los alumnos.
\end{abstract}

Palabras clave: enseñanza de lenguas extranjeras, fonética, TIC, FLE.

\section{Développement, analyse et évaluation de la production orale en cours de langue étrangère avec les nouvelles technologies}

\section{Résumé}

Dans cette étude expérimentale on présente des outils et des méthodes pour le développement des compétences de production orale chez des étudiants universitaires dans un cours de français langue étrangère. On procède à mesurer la production orale des apprenants à partir de l'analyse de la durée en temps réel des enregistrements sur podcasts, ainsi qu'à partir de l'opinion des apprenants dans une auto-évaluation de leur production orale. On propose plusieurs critères pour l'évaluation des productions orales en relation avec la correction de la prononciation (répétition, auto-réflexion, méthode, collaboration) tels qu'ils se manifestent dans les enregistrements audio des étudiants.

Mots clés: enseignement d’une langue étrangère, phonétique, TICE, FLE.

Development, analysis and evaluation of oral production in foreign language class with new technologies

\begin{abstract}
This experimental study presents tools and methods to develop proficiency in oral production among university students taking a course in French language. A measurement of oral production of learners
\end{abstract}


based on the analysis of the duration of Real-time recording podcasts, as well as on the estimate of students from a self-report of their oral production is presented. Several criteria are proposed for assessing oral production in relation to the correct pronunciation (repetition, self-reflection method, collaboration) being all collected in the audio recordings of students.

Key words: teaching of foreign languages, phonetics, ICT, FLE.

Sumario: 1. Introducción. 2. Material y métodos. 2.1. Participantes de la investigación. 2.2. Herramientas para la producción oral. 2.3. Encuestas de los alumnos. 2.4. Aspectos legales. 2.5. Plan de trabajo para la producción oral. 2.6. Métodos de corrección de la producción oral. 3. Resultados: Las producciones orales de los alumnos. 3.1. Medición y cuantificación. 3.2. Evaluación de la producción oral. 4. Conclusiones.

\section{Referencia normalizada}

Tomé Díez, M. (2015). "Desarrollo, análisis y evaluación de la producción oral en clase de lengua extranjera con las nuevas tecnologías”. Thélème. Revista Complutense de Estudios Franceses, Vol. 30, Núm. 2: 255-268. http://dx.doi.org/10.5209/rev_THEL.2015.v30.n2.48663

\section{Introducción}

El olvido o negligencia de la competencia de producción oral en la enseñanza de idiomas ha sido señalada por varios autores (Renard, 1979; Murphy, 1991; Lauret, 2007; Wachs, 2011). Generalmente, en una clase de lengua extranjera, el aprendizaje de la pronunciación y la práctica de la corrección fonética han quedado en un segundo plano frente a la lengua escrita, la gramática o el vocabulario. Aunque las directivas internacionales y nacionales para la enseñanza de idiomas indican la adquisición de competencias escritas y orales (Marco Común Europeo de Referencia para las lenguas) la producción oral no suele estar suficientemente desarrollada en los distintos contextos educativos.

Para tratar al menos de equilibrar en clase de idiomas el papel de la producción oral con el resto de competencias es necesario poder cuantificar o medir las distintas prácticas o actividades dedicadas a la misma. Esto podría llegar a realizarse cuando se dispone de una sala multimedia o un laboratorio de idiomas, pero resulta mucho más complejo controlar las producciones orales de los alumnos tanto en el aula de clase como en su trabajo personal no presencial.

No resulta menos compleja la posibilidad de evaluar la producción oral del alumno en la progresión del curso académico, además de tener en cuenta el factor de la corrección fonética en el proceso de aprendizaje de la pronunciación. La aplicación de tests o la ayuda de programas informáticos pueden proporcionar algunos resultados, como recogen varios autores (Swanson \& Early, 2008; Kamber \& Skupien-Dekens, 2010; Barker et al., 2011), si bien este es un campo apenas investigado en la enseñanza de idiomas. 
El propósito de este estudio es avanzar ante las anteriores dificultades y tratar de responder a las siguientes preguntas que guían la investigación:

- ¿ ¿Cómo potenciar e incrementar la producción oral de los alumnos en clase de francés lengua extranjera (FLE)?

- ¿ ¿Cómo medir y cuantificar la producción oral de los alumnos?

- ¿Cómo evaluar las producciones orales en relación con la corrección y mejora de la pronunciación de los alumnos?

\section{Material y métodos}

\subsection{Participantes de la investigación}

Los alumnos implicados en esta investigación forman parte de la asignatura Lengua Francesa I del Grado en Filología Moderna: Inglés de la Universidad de León, con una carga lectiva de 6 créditos ECTS, una media de 35 estudiantes, durante tres cursos académicos: de 2011 a 2014. Su nivel de francés es variable, si bien la mayor parte se sitúa en un nivel principiante o bien en "faux débutant". La asignatura se imparte en el segundo semestre del curso, entre los meses de febrero y junio, en una media de 3 horas semanales presenciales en el aula. Los manuales utilizados son Le Nouveau Taxi 1! (Capelle \& Menand, 2009) y Cahier de prononciation française (Duflot \& Tomé, 2005). Véase referencia a la Guía docente en la bibliografía.

\subsection{Herramientas para la producción oral}

Los términos podcast o podcasting (compuestos de Pod -de Apple iPod- y broadcast: transmisión, emisión, difusión) hacen referencia a un archivo de audio digital (generalmente en formato mp3), así como a la distribución mediante un sistema de redifusión (RSS). La utilización de podcasts en la enseñanza de idiomas ha sido abordada por varios autores (Hasan \& Hoon, 2013), si bien son pocos los investigadores que se han centrado en su aplicación al aprendizaje de la pronunciación o a la producción oral de los alumnos (Ducate \& Lomicka, 2009; Knight, 2010; Tomé, 2009, 2011).

Para la creación de podcasts los alumnos podían elegir entre las siguientes modalidades: A) grabadora web en red social (Audio Boo, Soundcloud, Twaud.io); B) grabadora del teléfono o dispositivo móvil; C) grabadora del ordenador (Windows, Audacity, Freecorder).

Estas grabaciones audio debían estar agrupadas en un espacio web de fácil acceso y consulta, como es el proporcionado por las plataformas o comunidades de intercambio de podcasts Audio Boo, Soundcloud o la ya desaparecida Twaud.io. Cada producción oral del alumno debía ser identificable con un título: Leçon 2 Exercice 1; 2CO L5 E1 ( = Segunda corrección Lección 5 Ejercicio 1); Taxi Leçon 8; COL Sofia \& Monica L3 E1 (= Colaboración alumnos Sofía y Mónica Lección 3 Ejercicio1); Laura Créations; Chanson Hector; Echauffement Estefanía, TL20 Avec EA, AC et E Final (= Taxi Leçon 20 avec Effort Articulatoire, Autocorrection et 
Echauffement Final). Véase aspecto de Espacio de producción oral Podcast Audio Boo de un alumno en Figura 1.
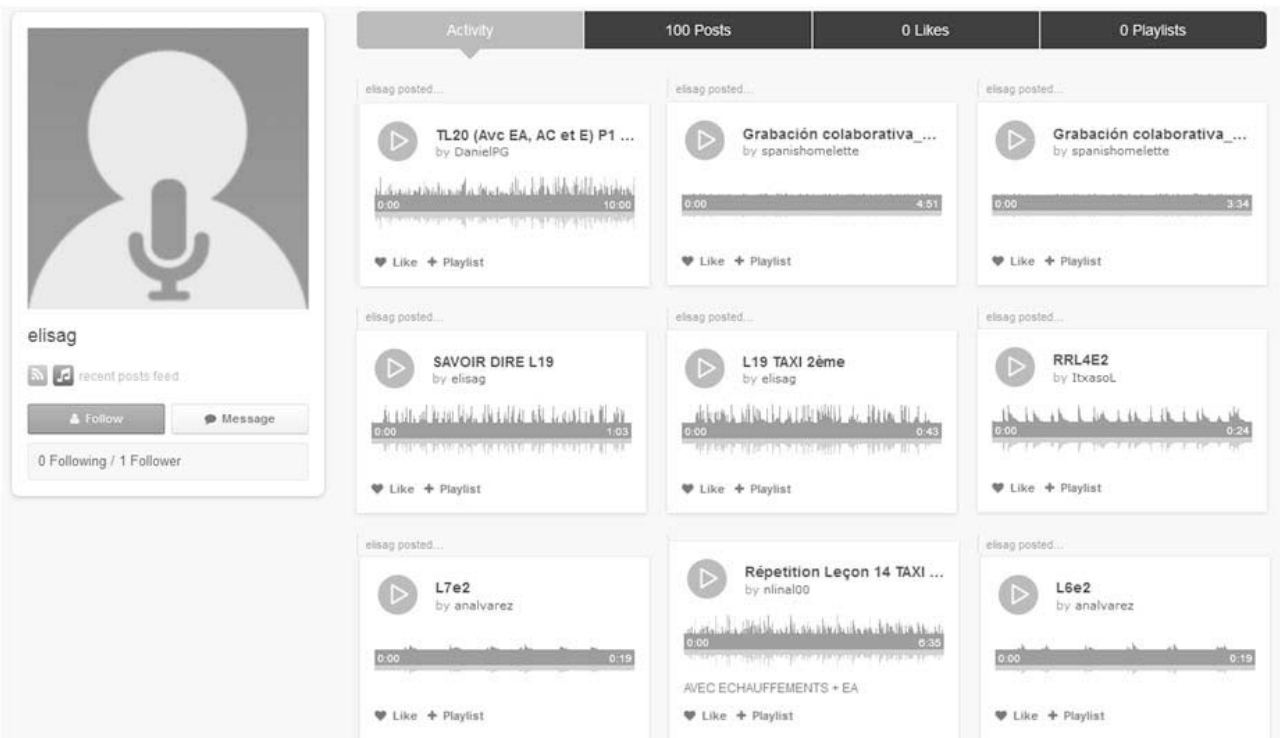

Figura 1: Espacio de producción oral en Podcast Audio Boo de un alumno

Las grabaciones audio de los alumnos están disponibles online dentro del Proyecto Oral FLE Prononciation, base de datos de las producciones orales de los estudiantes de francés lengua extranjera, que forma parte del Proyecto FLENET de la Universidad de León. En cada uno de los directorios siguiente se puede consultar la lista del conjunto de los podcasts de cada curso académico (véase webs tipo: WikiPodcastsCampusFLE_2012-2013.html), así como una selección de determinadas producciones orales que actualizan calentamientos vocales, refuerzos y entrenamientos, autocorrecciones, ruidos u onomatopeyas, canciones, teatralizaciones, correcciones colaborativas):

EtuPodcasts2012, URL: http://flenet.rediris.es/OralFLEprojet/TachesBlogs/EtuPodcasts2012/

EtuPodcasts2013, URL: http://flenet.rediris.es/OralFLEprojet/TachesBlogs/EtuPodcasts2013/

EtuPodcasts2014,

URL: http://flenet.rediris.es/OralFLEprojet/TachesBlogs/EtuPodcasts2014/ 


\subsection{Encuestas de los alumnos}

Se propone a los alumnos el siguiente autoinforme y cuestionario:

Autoinforme (self-report questionnaire) para la medición del tiempo dedicado a la producción oral, que consta de los apartados: A) Producción oral Empleo del tiempo en clase; B) Producción oral - Grabaciones audio - Podcast. El alumno debe anotar el tiempo estimado (minutos, segundos) de sus producciones orales, utilizando las distintas casillas que hacen referencia a meses y semanas. En el primer apartado se computan las intervenciones orales del alumno en las horas de clase presencial (respuestas, lecturas, consultas, etc.). En el segundo apartado se computa tanto el tiempo estimado en la preparación de las grabaciones audio, como el tiempo real de producción oral recogido en el espacio Podcast de cada alumno. Véase: Anexo 1: Autoinforme de la Producción Oral del alumno.

- Cuestionario de la opinión de los alumnos en torno a las prácticas y trabajos desarrollados durante el curso sobre el aprendizaje de la pronunciación y la corrección fonética. Véase: Anexo 2: Cuestionario Final Pronunciación Impresiones de los alumnos.

\subsection{Aspectos legales}

Para preservar los derechos a la intimidad de los alumnos en relación con sus grabaciones publicadas en redes sociales, se propone que sus nombres o apellidos no figuren en los espacios web creados; se indica la posibilidad de mantener sus podcasts en modo privado, al tiempo que se les informa de los riesgos o perjuicios ante la utilización de sus grabaciones por otros internautas. Se propone un acuerdo o Carta entre el docente y los alumnos que recoge estos aspectos así como el uso exclusivo de los podcasts para fines docentes o investigadores.

\subsection{Plan de trabajo para la producción oral}

Se propone al alumno la grabación de los siguientes tipos de ejercicios y tareas pedagógicas (Véase Anexo 3: Production Orale - Plan de travail):

Escucha y repetición o lectura de ejercicios del Cahier de prononciation française (Duflot \& Tomé, 2005). Cada ejercicio consta de pares de palabras o frases que actualizar una oposición fonológica o se centran en uno de los fonemas fundamentales del francés.

Lectura de textos del método de francés Le Nouveau Taxi 1! (Capelle \& Menand, 2009). Varios textos deberán se grabados aplicando la técnica del Effort Articulatoire (marcar o exagerar los fonemas), la Autocorrección (espontánea o teatralizada) u aplicando otros métodos de clase. El alumno deberá elaborar un esquema en su cuaderno que implica una preparación / reflexión centrada en los fonemas / dificultades a reforzar o corregir.

El docente tutoriza las producción oral de los alumnos, escuchando los podcasts, detectando los errores o fonemas que se deben reforzar e indicando la 
secuencia o ejercicio que debe ser repetido / corregido. Asimismo puede señalar la necesidad de practicar el Echauffement y la aplicación de los métodos propuestos en clase.

La repetición o corrección de un ejercicio o secuencia audio grabada por el alumno implica que debe ser indicada claramente en el título (Repetición / Corrección 1, L2 E1; Repetición / Corrección 2, L7 E2, etc.), así como ir precedida de la grabación de un Echauffement o una técnica de refuerzo del fonema en cuestión.

- Creaciones facultativas: el alumno puede aportar su creatividad grabando varios tipos de secuencias de producción oral: lecturas, canciones, teatralizaciones, juegos, etc., debiendo privilegiar la actualización de los métodos y técnicas propuestos en clase (Echauffement, Autocorrection, Effort articulatoire, etc.).

Grabaciones colaborativas: De forma facultativa o para ayudar a alumnos con dificultades, dos o tres alumnos pueden realizar producciones orales en colaboración, que podrían ser de dos tipos: A) Tutorización en la corrección de un ejercicio que debe ser repetido: el alumno monitor / tutor indica errores, refuerzos y técnicas a realizar por alumno con dificultades. B) Producciones orales creativas que actualicen o desarrollen los métodos y técnicas propuestos en clase (Echauffement, Autocorrection, Effort articulatoire, etc.).

\subsection{Métodos de corrección de la producción oral}

Los trabajos de Lyster \& Ranta (1997), Callamand (1981), Murphy (1991), Morris (2005), Engwall \& Bälter (2007) o Lauret (2007) hacen referencia a las distintas modalidades de corrección de la pronunciación tanto por parte del docente (explicit correction, recasts, clarification requests, metalinguistic feedback, elicitation, repetition) como por parte del alumno (repetition, incorporation, self-repair, peerrepair), así como a las múltiples técnicas de corrección fonética: repeticiones, refuerzos, calentamiento vocal, instrucciones lingüísticas, teatralizaciones, autocorrecciones, corrección colaborativa entre alumnos, etc.

Los intercambios entre el alumno, el docente u otros alumnos para la adquisición y la corrección de la pronunciación resultan determinantes. El docente o tutor debe detectar los errores fundamentales, al tiempo que estimular al alumno para que se corrija él mismo. El error no debe ser una fuente de bloqueo para el alumno, sino un motivo de juego, interacción o intercambio con el docente o con otros alumnos. La corrección de la pronunciación debe ser dinámica y tener un sentido real para motivar al alumno en la resolución de problemas y la colaboración con el docente u otros alumnos. Es esencial que el alumno descubra sus dificultades y reflexione sobre el proceso de corrección de su pronunciación.

Se deben tener en cuenta las aportaciones eficaces de los distintos métodos de corrección fonética, como el método articulatorio o el de oposiciones fonológicas, así como las múltiples técnicas y entornos que pueden favorecen la adquisición y práctica de la pronunciación (ejercicios, actividades, manuales, canciones, teatralizaciones, laboratorio multimedia, etc.). No obstante destacamos la 
importancia de los trabajos de Guberina (1965) y Renard (1979) sobre el método verbo-tonal de corrección fonética en aspectos como la lengua como instrumento de comunicación, la noción de criba fonológica, el sistema de faltas en el aprendizaje de cada lengua, la enseñanza de la fonética en situación de comunicación, tratamiento del error del alumno desde la prosodia, la fonética combinatoria, los contextos facilitadores y la pronunciación matizada. En relación con los alumnos principiantes (débutants) de francés lengua extranjera se aconseja abordar un sistema vocálico simplificado y no normativo (Companys, 1981; Leon, 1964; Tomé, 1994).

Los errores fundamentales de los alumnos de FLE españoles están relacionados tanto con factores suprasegmentales (ritmo y entonación en general) como segmentales. Entre estos últimos destacamos: las vocales [y], /OE/ y las nasales; las semivocales; las consonantes [v], [z], [R] y las fricativas palatales que se corresponden con las grafías “ch” y “j” (Tomé, 1994).

\section{Resultados: Las producciones orales de los alumnos}

\subsection{Medición y cuantificación}

Para medir el conjunto de la producción oral de cada alumno hemos utilizado dos criterios: a. Tiempo real de las grabaciones comprobable por la duración de los archivos audio o podcasts del alumno; b. Autoinforme de la producción oral del alumno. Recogemos a continuación los totales relacionados con tres modelos de alumno según la calificación final obtenida: aprobado, notable o sobresaliente, así como la media de las producciones por curso académico. Los tiempos estimados por los alumnos en el total de un curso académico varían entre 6 a 10 minutos de producción oral en clase, y entre 60 a 80 minutos en la preparación que precede a las grabaciones.

Alumno tipo Aprobado:

Total del tiempo de grabación en podcast: 46,96 minutos.

Total del tiempo de producción oral estimado por el alumno: 110 minutos.

Alumno tipo Notable:

Total del tiempo de grabación en podcast: 57,13 minutos.

Total del tiempo de producción oral estimado por el alumno: 148 minutos.

Alumno tipo Sobresaliente:

Total del tiempo de grabación en podcast: 85,02 minutos.

Total del tiempo de producción oral estimado por el alumno: 178 minutos.

Curso 2011-2012: Total de media por alumno grabación en podcast: 60 minutos. Curso 2012-2013: Total de media por alumno grabación en podcast: 63 minutos. Curso 2013-2014: Total de media por alumno grabación en podcast: 64 minutos. 


\subsection{Evaluación de la producción oral}

Para poder valorar aspectos cualitativos en la producción oral del alumno debemos centrarnos en los diferentes aspectos relacionados con la corrección de dicha producción, por parte del docente o por parte de un compañero de clase. Distinguiremos los siguientes criterios:

A. Repetición de ejercicios o secuencias propuesta por el docente tras la detección de errores de pronunciación. La necesidad de volver a grabar determinados ejercicios exige al alumno la identificación del error para su posterior corrección, una reflexión sobre la aplicación de estrategias de corrección, así como entrenamientos, calentamiento vocal o refuerzos que deben aparecer en la segunda o tercera grabación. Generalmente observamos que estas producciones orales de repetición o corrección suponen una mejora en los problemas o errores de articulación y muestran la adquisición de una correcta pronunciación. Ocasionalmente estas repeticiones pueden realizarse con la colaboración o ayuda de un compañero de clase, obteniéndose una grabación en forma de dialogo, en la que el alumno en funciones de tutor corrige o propone la repetición de ciertas secuencias.

B. Autorreflexión del alumno para descubrir dificultades y errores de pronunciación. Cuando un alumno debe repetir y corregir determinados ejercicios y secuencias pone en práctica la autorreflexión en la identificación de los problemas al tiempo que debe actualizar algunos de los métodos propuestos por el docente $\mathrm{u}$ otro compañero. Muchas de las grabaciones revelan este proceso puesto que se observan pasajes con calentamiento vocal, refuerzo de fonema, ruido u onomatopeya y autocorrecciones que permiten la superación y corrección del error en cuestión.

C. Aplicación de métodos o técnicas de corrección en autonomía o en colaboración. En el plan de trabajo se invita a los alumnos a la utilización de algunos métodos de fácil aplicación para favorecer la corrección de determinados errores de pronunciación. Asimismo se propone a los alumnos que dejen constancia de los mismos en el transcurso de las grabaciones orales. De este modo se obtienen numerosas producciones orales que actualizan el esfuerzo articulatorio realizado por el alumno mediante ejercicios de calentamiento vocal, exageración o refuerzo de un fonema, ruido u onomatopeya, autocorrección, canción o teatralización.

D. Corrección colaborativa entre alumnos. Las producciones orales que se realizan entre dos alumnos pueden ser creativas (diálogos, teatralizaciones, canciones) o de ayuda para la repetición y corrección de determinados fonemas. Estas implican una preparación y reflexión previas, la integración de estrategias de corrección adecuadas, así como una simulación de la interacción entre el docente y el alumno. Cuando los alumnos ejecutan este tipo de grabaciones se actualizan varios de los métodos propuestos por el docente, conduciendo normalmente a una excelente corrección y adquisición adecuada de la pronunciación.

Cuando todos estos índices están presentes en las grabaciones orales de los alumnos podemos decir que el proceso de corrección alcanza sus objetivos al 
tiempo que se revela como una herramienta y método eficaz que el alumno podrá aplicar en futuras formaciones, cursos, tareas o situaciones de comunicación real. Como revelan los resultados del Cuestionario Final de Pronunciación sobre las Impresiones de los alumnos, estos creen disponer de métodos y estrategias para corregir su propia pronunciación, así como estar en condiciones de enseñarlos o aplicarlos si tuvieran que desempeñar tareas docentes o de tutorización algún día.

\section{Conclusiones}

Creemos que es posible potenciar e incrementar la producción oral de los alumnos que estudian una lengua extranjera (FLE) si se utilizan herramientas adecuadas y eficaces como los podcasts que permiten la grabación de la voz de los alumnos en un entorno comunicativo e interactivo. Es importante que el plan de trabajo para la producción oral propuesto por el docente sea equilibrado, dinámico y abierto a la creatividad del alumno. La tutorización y acompañamiento del docente, del tutor o de los propios alumnos es imprescindible en el proceso de adquisición y corrección de la pronunciación.

Para medir y cuantificar la producción oral de los alumnos hemos utilizado dos criterios complementarios, uno objetivo y fiable (tiempo real de duración de las grabaciones en podcasts) y el otro subjetivo y aproximado (autoinforme de la producción oral del alumno). Los resultados globales arrojan una media en torno a los 150 minutos de producción oral por alumno durante un curso académico que comprende cuatro meses. Resulta difícil contrastar y valorar estos resultados ya que no disponemos de investigaciones de medición de la producción oral en la enseñanza de una lengua extranjera hasta la actualidad. No obstante somos optimistas ante el resultado obtenido, especialmente si lo ponemos en relación con los minutos que representa el tiempo de producción oral por alumno y curso académico, durante las 3 horas de clase semanales: de 6 a 10 minutos.

Finalmente hemos podido evaluar las producciones orales en relación con la corrección y mejora de la pronunciación de los alumnos. Para lo cual hemos tenido que conjugar aspectos cuantitativos (medición de la producción oral) y cualitativos (repeticiones, autocorrecciones, métodos, colaboraciones, plan de trabajo). Debemos destacar la importancia de las grabaciones como elemento determinante para el registro y detección de errores tanto por parte del docente como del propio alumno que puede así escuchar de nuevo y contrastar su producción oral. Otros elementos decisivos en el proceso de aprendizaje del alumno son la práctica de la autorreflexión, la aplicación de estrategias en contextos reales y la creación de correcciones colaborativas. La opinión de los alumnos a través del cuestionario valora positivamente los descubrimientos adquiridos, los métodos de corrección aplicados y el desarrollo de la autorreflexión y conciencia del error. Así pues hemos podido observar en las distintas fases del proceso una mejora y perfeccionamiento de la pronunciación (adquisición de fonemas problemáticos, fluidez, ritmo y entonación) y, lo que es más importante, todos estos índices o pruebas (ejercicios de calentamiento vocal, refuerzo de fonemas, ruidos u onomatopeyas, autocorrecciones, 
teatralizaciones) han quedado recogidos en las grabaciones de los podcasts de los alumnos.

\section{REFERENCIAS BIBLIOGRÁFICAS}

Barker, F. (et al.), (2011) "Identifying criterial aspects of pronunciation in L2 English across CEFR levels: Implications for language learning" in Angouri, J., Daller, M. \& J. Treffers-Daller (eds.), The Impact of Applied Linguistics: Proceedings of the 44th Annual Meeting of BAAL. University of the West of England, Scitsiugnil Press, pp. 17-22. Disponible en: http://www.baal.org.uk/proceedings_11.pdf [Último acceso el 5 de septiembre de 2015].

Callamand, M., (1981) Méthodologie de l'enseignement de la prononciation. Paris, Clé International.

Capelle, G., \& R. Menand, (2009) Le Nouveau Taxi ! 1. Méthode de français. Paris, Hachette.

Companys, M., (1981) Méthodologie de l'enseignement de la prononciation. Paris, Clé International.

Ducate, L. \& L. Lomicka, (2009) "Podcasting: An Effective Tool for Honing Language Students' Pronunciation?” in Language Learning \& Technology [En línea]. Vol. 13, $\quad \mathrm{n}^{\circ} 3, \quad$ disponible http://lt.msu.edu/vol13num3/ducatelomicka.pdf [Último acceso el 5 de septiembre de 2015], pp. 66-86.

Duflot, C. \& M. Tomé, (2005) Cahier de prononciation française. Exercices pour les étudiants espagnols. León, Universidad de León. Disponible en: http://flenet.unileon.es/phon/cahier/ [Último acceso el 5 de septiembre de 2015].

Engwall, O. \& O. Balter, (2007) "Pronunciation Feedback from Real and Virtual Language Teachers" in Computer Assisted Language Learning. Vol. 20, $\mathrm{n}^{\circ} 3$, Julio 2007, pp. 235-262(28).

Guberina, P., (1965) "La méthode audio-visuelle structuro-globale" in Revue de Phonétique Appliquée, Université de Mons. № 1, pp. 35-64.

Guía docente de la asignatura Lengua Francesa I de la Universidad de León; Código 00413010.

Disponible

en:

https://guiadocente.unileon.es/docencia/guia_docent/doc/asignatura.php?assignat ura $=0413010 \& a n y \_$academic $=2013 \_14 \&$ idioma $=$ cast \&doc $=\mathrm{N} \#$ [Último acceso el 5 de septiembre de 2015].

Hasan, M. \& T. Hoon, (2013) "Podcast Applications in Language Learning: A Review of Recent Studies" in English Language Teaching [En línea]. Vol. 6, n 2, disponible en : http://www.ccsenet.org/journal/index.php/elt/article/viewFile/23820/15118 [Último acceso el 5 de septiembre de 2015].

Kamber, A. \& C. Skupien-Dekens, (2010) "La correction phonétique en français langue étrangère: enseignement et évaluation en laboratoire multimédia" in Cahiers de l'APLIUT [En línea]. Vol. XXIX, $\mathrm{n}^{\circ} 2$, disponible en : http://apliut.revues.org/744 [Último acceso el 5 de septiembre de 2015]. 
Knight, R., (2010) "Sounds for Study: Speech and Language Therapy Students: Use and Perception of Exercise Podcasts for Phonetics" in International Society for Exploring Teaching and Learning. Vol. 22, $\mathrm{n}^{\circ}$ 3, pp. 269-276.

Lauret, B., (2007) Enseigner la prononciation: questions et outils. Paris, Hachette.

Leon, P. \& M. Leon, (1964) Introduction à la phonétique corrective. Paris, Hachette / Larousse.

Lyster, R. \& L. Ranta, (1997) “Corrective feedback and learner uptake” in Studies in Second Language Acquisition [En línea]. Vol. 19, disponible en: http://people.mcgill.ca/files/roy.lyster/Lyster_Ranta1997_SSLA.pdf [Último acceso el 5 de septiembre de 2015], pp. 37-66.

Morris, F., (2005) "Child to child interaction and corrective in a computer mediated L2 class" in Language Learning and Technology [En línea]. Vol. 9, $\mathrm{n}^{\circ} 1$, disponible en: http://lt.msu.edu/vol9num1/morris/default.html [Último acceso el 5 de septiembre de 2015], pp. 29-45.

Murphy, J., (1991) "Oral communication in TESOL: Integrating speaking, listening and pronunciation" in TESOL Quarterly. Vol. 25, n 1, pp. 51-75.

Renard, R., (1979) La méthode verbo-tonale de correction phonétique. Paris et Mons, Didier et CIPA.

Swanson, P. \& P. Early, (2008) "Digital recordings and assessment: An alternative for measuring oral proficiency" in Moeller, A., Theiler, J. \& S. Betta (Eds.), CSCTFL Report. Eau Claire, WI, Central States Conference on the Teaching of Foreign Languages, pp. 129-143. Disponible en: http://digitalarchive.gsu.edu/mcl_facpub/3/ [Último acceso el 5 de septiembre de 2015].

Tomé, M., (1994) Fonética francesa para uso de estudiantes españoles. León, Universidad de León.

Tomé, M, (2009) "Productions orales, weblogs et projet de télécollaboration avec le web 2.0 pour l'enseignement du français (FLE)" in Revue ALSIC [En línea]. Vol. 12, disponible en : http://alsic.revues.org/index1279.html [Último acceso el 5 de septiembre de 2015], pp. 90-108.

Tomé, M., (2011) "Réseaux et médias sociaux sur internet pour l'apprentissage de la prononciation d'une langue étrangère" in International Journal of E-Learning \& Distance Education [En línea]. Vol. 25, $\mathrm{n}^{\circ} 2$, disponible en : http://www.jofde.ca/index.php/jde/article/view/724/1261 [Último acceso el 5 de septiembre de 2015].

Wachs, S., (2011) "Tendances actuelles en enseignement de la prononciation du français, langue étrangère (FLE)” in Revista de Lenguas Modernas [En línea]. $\mathrm{N}^{\circ}$ 14, disponible en : http://revistas.ucr.ac.cr/index.php/rlm/article/view/9687 [Último acceso el 5 de septiembre de 2015], pp. 183-196. 


\section{ANEXOS}

Anexo 1: Autoinforme de la Producción Oral del alumno:

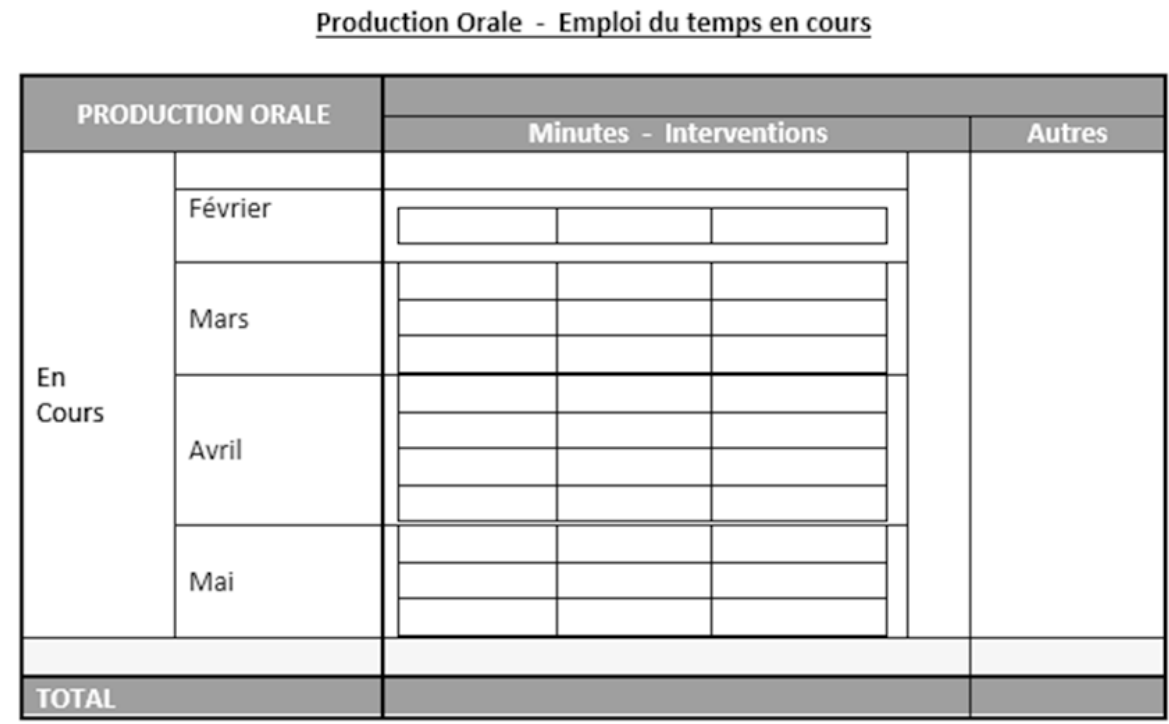

$\underline{\text { Production Orale - Enregistrements Audio - Podcasts }}$

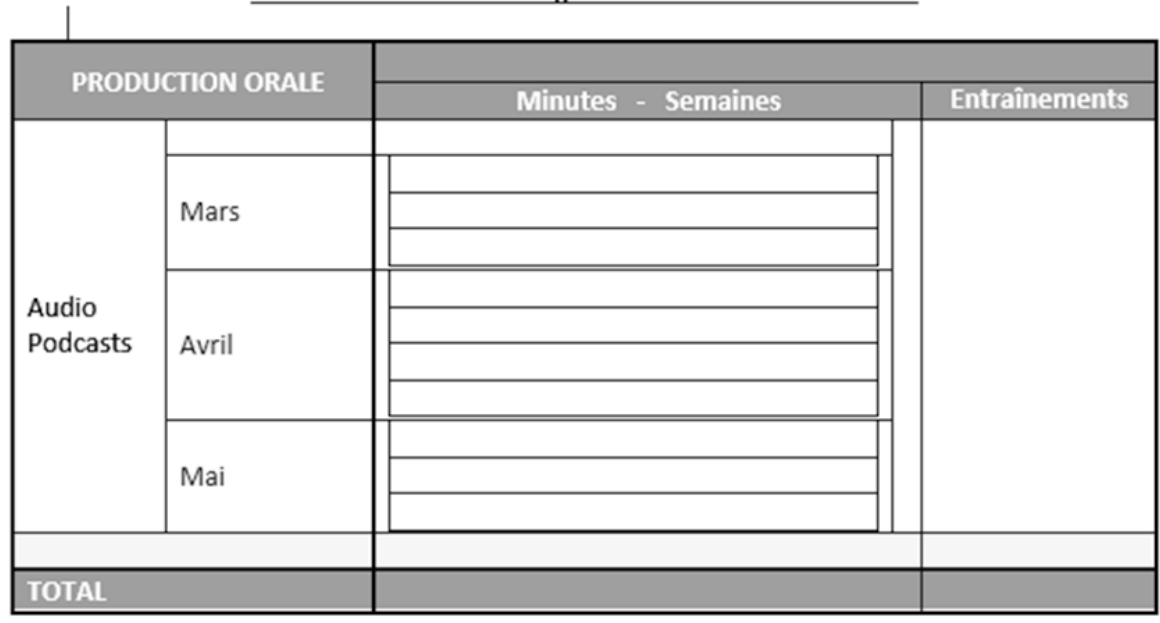




\section{Anexo 2: Cuestionario Final Pronunciación - Impresiones de los alumnos}

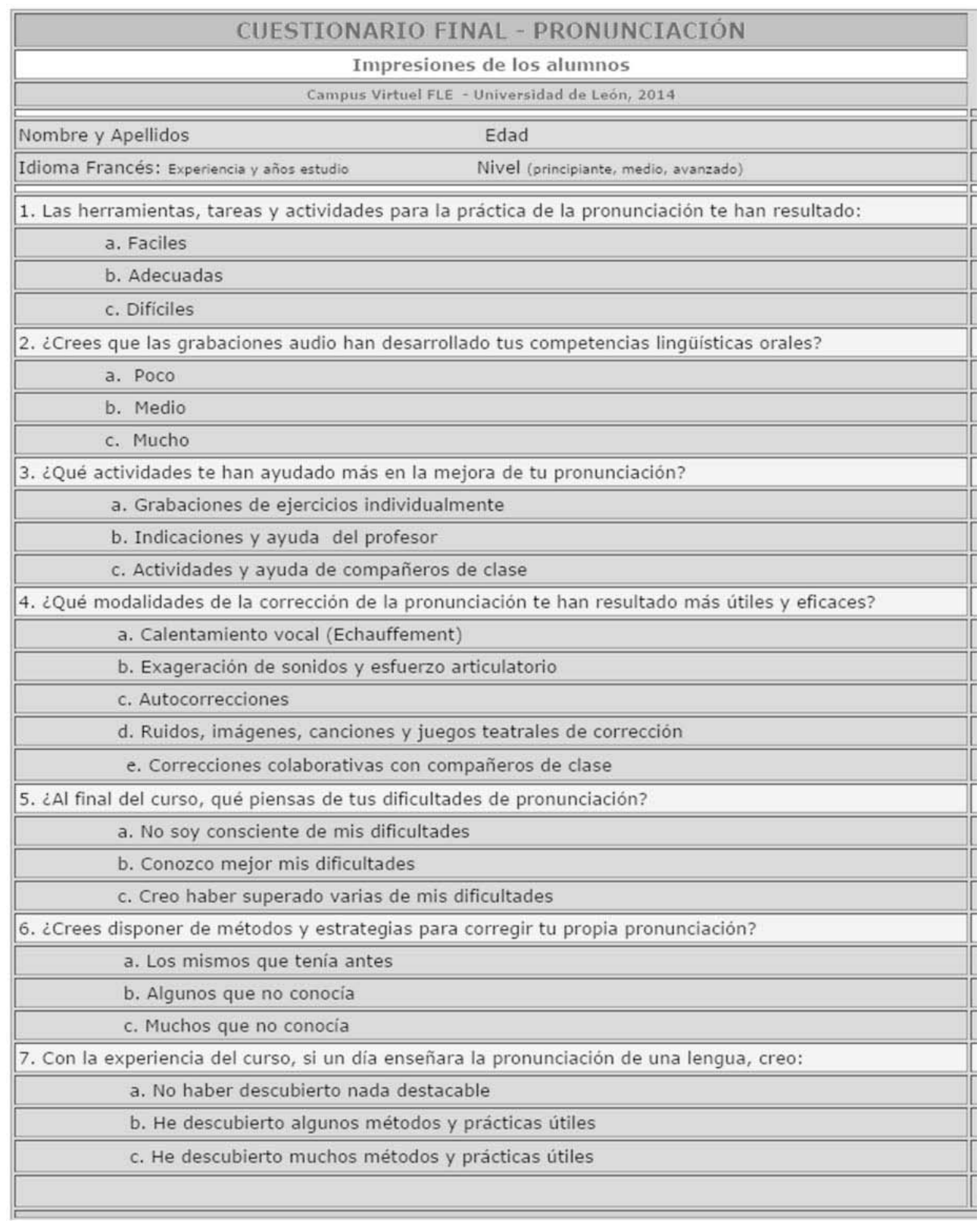


Anexo 3: PRODUCTION ORALE - PLAN DE TRAVAIL:

Semana 1: Leçon 1 Exercice, 1 Leçon 2 Ex. 1, Leçon 3 Ex. 1, Texte Taxi L1 Taxi L 2

S2: Leçon 3 2ème Partie Ex. 1 L.4 Ex. 1. L5 Ex. 1 L6 Ex. 1 L7 Ex. 1 L8 Ex. 1 L9 Ex. 1

S3: CORRECTIONS (indiquant R L1 E1) avec Echauffement / Méthode ; L1 E2 L2 E2 - L3 E2 - L32P E2 - L4 E2 - L5 E2

S4: L6 E2 - L7 E2 - L8 E2 - L9 E2 - CORRECTIONS (indiquant R L1 E1) avec Echauffement - Méthode.

S5: CORRECTIONS + Echauffement enregistré; identifiez et notez les erreurs de prononciation dans votre cahier. Textes Taxi: Leçons 5, 6 et 7 - Créations facultatives: chansons, onomatopées sur les phonemes.

S6: CORRECTIONS + Echauffement enregistré; identifiez et notez les erreurs de prononciation dans votre cahier. Textes Taxi: Leçons 9, 10 et 11 avec effort articulatoire.

S7: L1 Ex.3, L2 Ex.3, L31P Ex. 3, L3 2P Ex. 3, L 4 Ex.3 + Echauffement enregistré. Textes Taxi: Leçons 13 et $14+$ préparation/réflexion dans votre cahier.

S8: L5 Ex.3, L6 Ex.3, L7 Ex. 3, L8 EX. 3, L 9 Ex.3 + Echauffement enregistré. Textes Taxi: Leçons 14 et 15 + préparation/réflexion dans votre cahier. Créations facultatives ou collaboratives.

S9: L1 Ex.4, L2 Ex 4, L31P Ex. 4, L3 2P Ex. 4, L 4 Ex.4 + Echauffement enregistré. Textes Taxi: Leçons 16 et 17 + préparation/réflexion dans votre cahier. CORRECTIONS (indiquant dans le titre Répetition 1 ou 2) avec Echauffement / Méthode. Créations facultatives ou collaboratives.

S10: L5 Ex.4, L6 Ex.4, L7 Ex. 4, L8 EX. 4, L 9 Ex.4 + Echauffement enregistré. Textes Taxi: Leçons 18 et 19 + préparation/réflexion dans votre cahier. CORRECTIONS (indiquant dans le titre Répetition 1 ou 2) avec Echauffement / Méthode. Créations facultatives ou collaboratives. 